\title{
Stepwise differentiation and functional characterization of human induced pluripotent stem cell-derived choroidal endothelial cells
}

Kelly Mulfaul 1,2, Joseph C. Giacalone ${ }^{1,2}$, Andrew P. Voigt ${ }^{1,2}$, Megan J. Riker ${ }^{1,2}$, Dalyz Ochoa ${ }^{1,2}$, Ian C. Han ${ }^{1,2}$, Edwin M. Stone ${ }^{1,2}$, Robert F. Mullins ${ }^{1,2}$ and Budd A. Tucker ${ }^{1,2^{*}}$

\begin{abstract}
Background: Endothelial cells (ECS) are essential regulators of the vasculature, lining arteries, veins, and capillary beds. While all ECs share a number of structural and molecular features, heterogeneity exists depending on their resident tissue. ECs lining the choriocapillaris in the human eye are lost early in the pathogenesis of age-related macular degeneration (AMD), a common and devastating form of vision loss. In order to study the mechanisms leading to choroidal endothelial cell (CEC) loss and to develop reagents for repairing the choroid, a reproducible in vitro model, which closely mimic CECs, is needed. While a number of protocols have been published to direct induced pluripotent stem cells (iPSCs) into ECs, the goal of this study was to develop methods to differentiate iPSCs into ECs resembling those found in the human choriocapillaris specifically.

Methods: We transduced human iPSCs with a CDH5p-GFP-ZEO lentiviral vector and selected for transduced iPSCs using blasticidin. We generated embryoid bodies (EBs) from expanded IPSC colonies and transitioned from $\mathrm{mTESR}^{\mathrm{TM}} 1$ to EC media. One day post-EB formation, we induced mesoderm fate commitment via addition of BMP-4, activin A, and FGF-2. On day 5, EBs were adhered to Matrigel-coated plates in EC media containing vascular endothelial cell growth factor (VEGF) and connective tissue growth factor (CTGF) to promote CEC differentiation. On day 14, we selected for CECs using either zeocin resistance or anti-CD31 MACS beads. We expanded CECs postselection and performed immunocytochemical analysis of CD31, carbonic anhydrase IV (CA4), and RGCC; tube formation assays; and transmission electron microscopy to access vascular function.

Results: We report a detailed protocol whereby we direct iPSC differentiation toward mesoderm and utilize CTGF to specify CECs. The CDH5p-GFP-ZEO lentiviral vector facilitated the selection of iPSC-derived ECs that label with antibodies directed against CD31, CA4, and RGCC; form vascular tubes in vitro; and migrate into empty choroidal vessels. CECs selected using either antibiotic selection or CD31 MACS beads showed similar characteristics, thereby making this protocol easily reproducible with or without lentiviral vectors.

(Continued on next page)
\end{abstract}

\footnotetext{
* Correspondence: Budd-Tucker@uiowa.edu

${ }^{1}$ Department of Ophthalmology and Visual Sciences, The University of lowa Carver College of Medicine, lowa City, IA 52242, USA

${ }^{2}$ Institute for Vision Research, The University of lowa, lowa City, IA 52242, USA
}

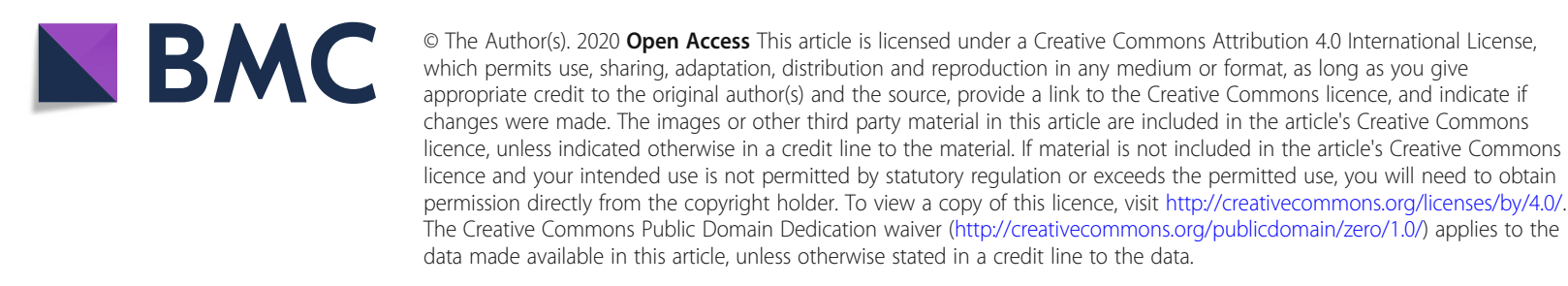


(Continued from previous page)

Conclusion: ECs generated following this protocol exhibit functional and biochemical characteristics of CECs. This protocol will be useful for developing in vitro models toward understanding the mechanisms of CEC loss early in AMD.

Keywords: Induced pluripotent stem cells (iPSCs), Choroid, Choroidal endothelial cells (CECs), Age-related macular degeneration (AMD), Connective tissue growth factor (CTGF)

\section{Background}

Endothelial cells (ECs) line the walls of blood vessels throughout the body, forming the innermost monolayers of vascular tubes. ECs are essential for facilitating the passage of oxygen and vital nutrients from the circulating blood to nourish tissue and organs, while removing $\mathrm{CO}_{2}$ and other waste products, thus maintaining tissue homeostasis. Under normal conditions, the EC barrier helps to restrict immune cell infiltration and activation. However, during times of injury, ECs can upregulate secreted and cell surface signals to greatly increase immune cell recruitment and promote an inflammatory response. Importantly, ECs can also aid in tissue repair by triggering blood vessel growth via angiogenesis. Regardless of their location in the body, ECs in any organ system share these basic functions. As such, essentially all ECs express a common set of marker genes including CD34, CD31, and von Willebrand's factor ( $v W F)$ [1]. Depending on their anatomical location, however, there is huge diversity in EC structure and function [2], including the expression of tissue-specific genes. For example, ECs in the arterial system express NOTCH4 and EFNB2 whereas ECs in the venous system express $N R 2 F 2$ and EphB4 [3]. Further molecular differences exist between ECs in different organs. Given the variability in endothelial cell subtypes, studying endothelial cells in vitro that are phenotypically similar to those from the relevant tissue is of high interest.

The choroid is a highly specialized vascular network, located between the neural retina and the sclera at the posterior pole of the eye. The choroid develops from the peri-ocular mesenchyme during embryonic development. Fate mapping in mice demonstrates that choroidal endothelial cells are derived from the mesoderm whereas other cell types within the choroid are derived from the neural crest [4]. The choroidal vasculature is essential for supporting healthy vision by providing nutrients to the retinal pigment epithelium (RPE) and photoreceptors while also removing waste products secreted by the RPE. The innermost layer of the choroid closest to the retina is a dense network of large-diameter capillaries, with a lobular arrangement, termed the choriocapillaris. The capillary walls are lined with specialized ECs, which have large fenestrations that allow for diffusion of nutrients, oxygen, and small proteins from the systemic circulation toward the retina, and removal of waste products from the RPE for systemic recycling. The choriocapillaris is supplied by medium-size arterioles that branch off of short posterior ciliary arteries and is drained through a confluence of venules in the vortex vein system near the equator of the eye [5-7].

Loss of choriocapillaris vessels occurs early in the pathogenesis of age-related macular degeneration (AMD). Immunohistochemical and gene expression analyses of human donor eyes demonstrate that endothelial cells lining the choriocapillaris are lost prior to RPE degeneration, creating empty lumens of extracellular matrix termed ghost vessels $[8,9]$. Morphometric analysis of the choroidal vasculature in eyes with AMD further supports choriocapillaris vessel loss in early AMD, but similar differences are not readily apparent in the deeper choroidal vessels, suggesting vascular dropout may occur specifically in the capillaries [10]. Standard methods of visualizing choroidal vascular anatomy in vivo include indocyanine green angiography, but this test is invasive, requiring an intravenous injection of dye [11, 12]. Recent advances in clinical imaging have allowed for non-invasive visualization of blood flow in the choroid in vivo and can be used to quantify vessel density. For example, studies using optical coherence tomography angiography (OCTA) have shown reduced vascular density in the choroidal vasculature of AMD patients compared to controls [13]. Most recently, swept-source OCTA, which provides improved visualization of choroidal anatomy, suggests that there are significant decreases in perfusion in the choriocapillaris in early AMD [14] as well as in advanced disease with geographic atrophy $[15,16]$. While these in vivo imaging modalities currently do not have the resolution to evaluate endothelial cell loss, the visualized differences in flow could be accounted for by the presence of empty choroidal vessels and are supported by the observation of increased fibrosis and increased ratio of stroma to vessels in AMD eyes by OCTA [17].

Although a number of published protocols have described differentiation of ECs from pluripotent embryonic stem cells [18-21], differences between EC subtypes have revealed the need for refined procedures suitable for directing tissue-specific EC commitment [3, 22]. We have previously shown that connective tissue growth factor (CTGF), when implemented during 
spontaneous differentiation, plays a key role in driving induced pluripotent stem cells (iPSCs) toward a choroidal endothelial cell (CEC) fate [23]. In the current report, we adopt a directed stepwise differentiation approach using bone morphogenetic protein 4 (BMP-4), fibroblast growth factor 2 (FGF2), activin A, and vascular endothelial growth factor (VEGF) [20], to enrich for mesoderm precursor cells prior to CEC specification using CTGF. This method, which was repeated using iPSCs generated from 3 independent individuals, provides a robust protocol for consistently producing iPSC-derived CECs.

\section{Methods}

The following protocol describes the stepwise differentiation of iPSCs into choroidal endothelial cells as summarized in Fig. 1. For in-depth, step-by-step instructions, see Additional file 1.

\section{Lentiviral production}

CDH5p-GFP-ZEO lentiviral vector was constructed incrementally. First, the CDH5 promoter was cloned into a pENTR5'-TOPO vector as previously demonstrated [24]. Then, GFP-T2A-Zeocin was amplified from pGreenZEO (System Biosciences) and subcloned into pENTR/D-TOPO. Finally, the pENTR5'CDH5p and pENTR/D-GFP-ZEO were cloned into the pDEST R4R3
Vector II (Thermo Fisher Scientific) using Gateway ${ }^{\text {ma }}$ LR Clonase $^{\text {Tm }}$ II Enzyme mix (Thermo Fisher Scientific). Note that the pDEST R4R3 Vector II includes a blasticidin resistance cassette for selection. The resulting vector was submitted to the Viral Vector Core Facility at the University of Iowa for lentiviral packaging. The CDH5pGFP-ZEO lentiviral vector is available on Addgene (cata$\log \#$ 122970). Figure 1b illustrates a simplified plasmid map.

\section{Viral transduction of iPSCs}

Three independent human iPSC lines were maintained in $\mathrm{mTeSR}^{\mathrm{rm}} 1$ (STEMCELL Technologies, USA) base media containing $5 \times$ supplement and primocin $(100 \mu \mathrm{g} /$ $\mathrm{ml}$ ) on Matrigel-coated six-well tissue culture plates. iPSC colonies were incubated with dispase (Thermo Fisher Scientific) at $37^{\circ} \mathrm{C}$ for $4-8 \mathrm{~min}$. Dispase was subsequently removed, and iPSC colonies were gently dislodged using $\mathrm{mTeSR}^{\mathrm{rm}} 1$. Cells were subsequently counted and plated at a density of 50,000 cells per well of a 24well plate. The next day, medium was replaced with $\mathrm{mTeSR}^{\mathrm{mw}} 1$ containing CDH5p-GFP-ZEO lentiviral particles for a final multiplicity of infection (MOI) of 10 (i.e., 10 viral particles per cell). After $48 \mathrm{~h}$, media containing lentiviral particles were replaced with $\mathrm{mTeSR}^{\mathrm{mw}} 1$ containing blasticidin $(5 \mu \mathrm{g} / \mathrm{ml})$. iPSCs were maintained in

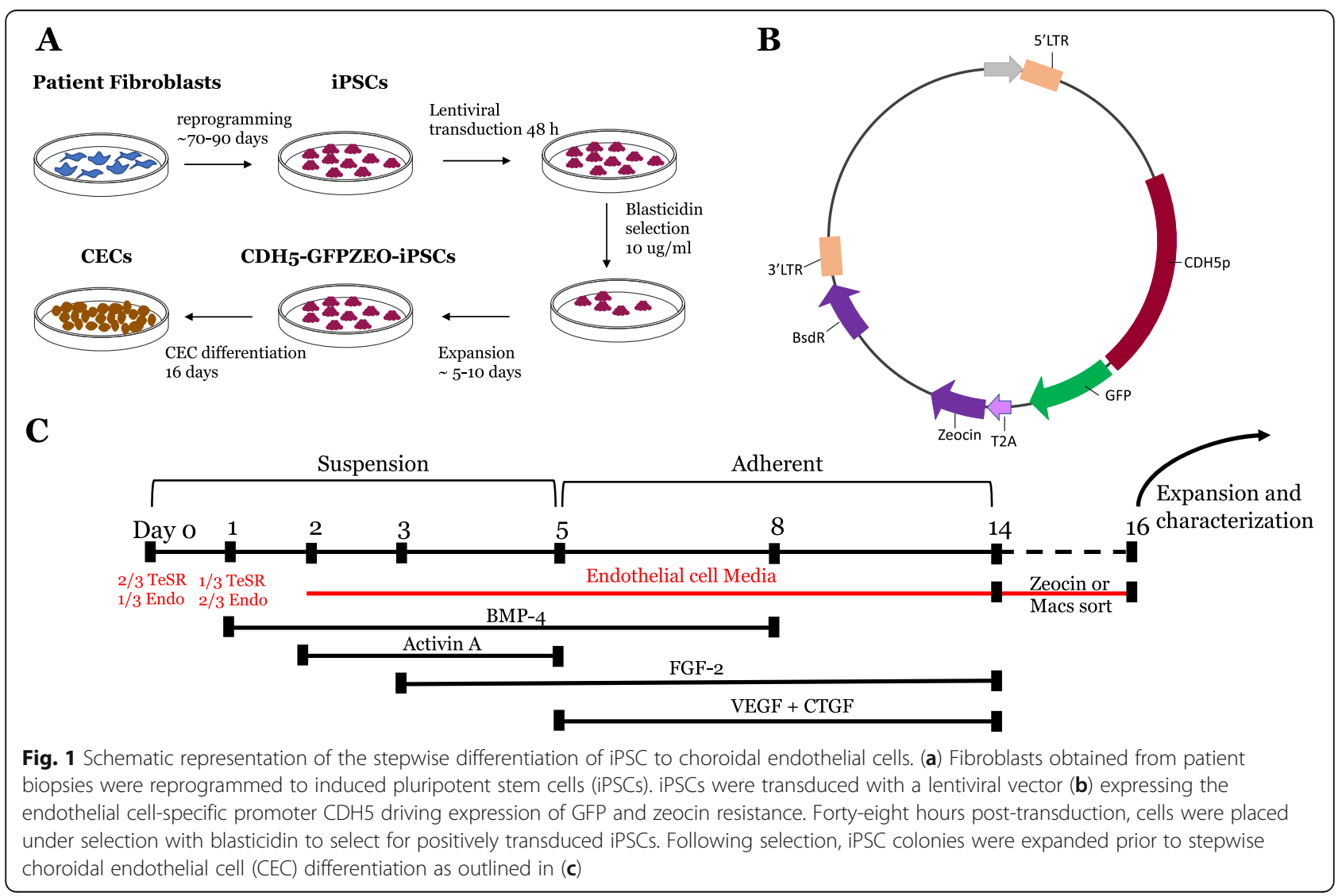


$\mathrm{mTeSR}^{\mathrm{Tw}} 1$ containing blasticidin $(5 \mu \mathrm{g} / \mathrm{ml})$ for $5-7$ days, changing medium daily.

\section{Embryoid body (EB) formation}

When iPSC colonies reached $80 \%$ confluency, colonies were dissociated using dispase (Thermo Fisher Scientific) at $37^{\circ} \mathrm{C}$ for $4-8 \mathrm{~min}$ or until the edges of the iPSC colonies began to curl. Dispase was subsequently removed, and iPSCs were gently lifted in DMEM taking care to prevent colonies from breaking up. iPSC colonies were allowed to settle by gravity in a $15-\mathrm{ml}$ conical tube for $5-10 \mathrm{~min}$ before resuspension in $6 \mathrm{ml} \mathrm{mTESR}{ }^{\mathrm{m}}{ }^{\mathrm{m}} 1$ (STEM CELL Technologies, USA) and $3 \mathrm{ml}$ endothelial cell growth media (R\&D systems). iPSCs were transferred to a $60-\mathrm{mm}$ cell culture dish pre-coated with polyHEMA (Sigma-Aldrich). PolyHEMA prevents iPSC colony adhesion. We refer to EB formation as day 0 of differentiation (Fig. 1c).

\section{EB-based differentiation of iPSC-derived choroidal endothelial cells}

On day 1 after EB formation, medium is replaced with $3 \mathrm{ml}$ of $\mathrm{mTeSR}^{\mathrm{im}} 1$ and $6 \mathrm{ml}$ endothelial cell media supplemented with $20 \mathrm{ng} / \mathrm{ml}$ BMP-4 (Miltenyi Biotec). On day 2, media were replaced with $9 \mathrm{ml}$ of endothelial cell media (R\&D systems) supplemented with $20 \mathrm{ng} / \mathrm{ml}$ BMP-4 (Miltenyi Biotec) and $10 \mathrm{ng} / \mathrm{ml}$ activin $\mathrm{A}$ (R\&D systems). On day 3, EB media were replaced $9 \mathrm{ml}$ of endothelial cell media supplemented with $20 \mathrm{ng} / \mathrm{ml}$ BMP-4 (Miltenyi Biotec), $10 \mathrm{ng} / \mathrm{ml}$ activin A, and $8 \mathrm{ng} / \mathrm{ml} \mathrm{FGF-2} \mathrm{(R \& D} \mathrm{systems).} \mathrm{After} 2$ days (day 5), EBs were collected and adhered, by gravity, to wells of a 6-well cell culture plate pre-coated with Matrigel at a density of 30 EBs per well in endothelial cell media supplemented with 10 ng/ml BMP-4, 8 ng/ml FGF-2, 25 ng/ml VEGFa (Peprotech), and $25 \mathrm{ng} / \mathrm{ml}$ CTGF (Peprotech). On day 6, media were replaced with fresh endothelial cell media supplemented with $10 \mathrm{ng} / \mathrm{ml}$ BMP-4, $8 \mathrm{ng} / \mathrm{ml}$ FGF-2, $25 \mathrm{ng} / \mathrm{ml}$ VEGFa, and 25 $\mathrm{ng} / \mathrm{ml}$ CTGF. After 2 days, on day 8 , media were replaced with endothelial cell media supplemented with $8 \mathrm{ng} / \mathrm{ml} \mathrm{FGF-}$ 2, $25 \mathrm{ng} / \mathrm{ml}$ VEGFa, and $25 \mathrm{ng} / \mathrm{ml}$ CTGF. Cells were maintained in these media from days 8-14, refreshing media every other day. A schematic summary of media changes is presented in Fig. 1c.

\section{Positive selection for endothelial cell population}

\section{A) Zeocin selection}

On day 14, differentiating cells were placed under antibiotic selection in endothelial cell media (R\&D systems) containing $25 \mathrm{ng} / \mathrm{ml}$ of zeocin (Thermo Fisher Scientific) for $48 \mathrm{~h}$.

OR

\section{B) CD31 microbead MACS sort}

Alternatively, on day 14, differentiating cells can be positively enriched for CD31-positive endothelial cells using CD31 MACS microbeads. Single cell suspensions were prepared using TrypLE (Thermo Fisher Scientific). Cells were pelleted by centrifugation and resuspended in PBS to a final concentration of $1 \times 10^{7} \mathrm{cell} / \mathrm{ml}$. Twenty microliters of FCR blocking agent (Miltenyi Biotech) was added per $60 \mu \mathrm{l}$ of cells as per the manufacturer's instructions, followed by $20 \mu \mathrm{l}$ of CD31 beads (Miltenyi Biotech) per $60 \mu \mathrm{l}$ of cells. Cells were incubated with the $\mathrm{CD} 31$ beads at $4{ }^{\circ} \mathrm{C}$ for $15 \mathrm{~min}$ with gentle shaking. One milliliter of PBS was added to the cell-bead suspension which was then pelleted by centrifugation. The pellet was resuspended in $1 \mathrm{ml}$ of PBS containing 0.04\% nonacetylated BSA. Cells were sorted on an autoMACS sorter using the selection program "possel." The positive fraction was plated into one well of a 6-well plate precoated with Matrigel and maintained in endothelial cell media (R\&D systems).

\section{Endothelial cell characterization}

A total of 50,000 cells were plated per well of an 8-well glass chamber slide or a 24-well plate. Endothelial cell media (R\&D) were changed every other day until cells reached the desired confluency. Media were aspirated and cells were fixed with $4 \%$ PFA for $10 \mathrm{~min}$ at room temperature. Cells were blocked in 5\% normal goat serum (NGS), 3\% bovine serum albumin (BSA), and $0.05 \%$ Triton X-100 for $1 \mathrm{~h}$ at room temperature and incubated with anti-CD31 (Abcam ab32457), anti-CA4 (R\&D Systems MAB21861), or anti-RGCC (Developmental Studies Hybridoma Bank PCRP-RGCC-2C4) in blocking buffer overnight at $4{ }^{\circ} \mathrm{C}$. Cells were washed 3 times with $\mathrm{PBS}$ and incubated with donkey anti-mouse 546 (Invitrogen A10036) or donkey anti-rabbit 546 (Invitrogen A10040) for $2 \mathrm{~h}$ at room temperature, washed 3 times, and counterstained with DAPI. Cells in 24-well plates were analyzed using epifluorescence (EVOS FL; AMG Life Technology). Cells grown on chamber slides were analyzed using confocal microscopy.

\section{Acetylated LDL uptake}

A total of 50,000 ECs were plated per well of a 24-well Matrigel-coated culture dish. Twenty-four hours later, $10 \mu \mathrm{g} / \mathrm{ml}$ of Alexa 488-labeled acetylated LDL (Thermo Fisher Scientific L233380) was added per well. ECs were incubated with acetylated LDL for 2-4 h. LDL uptake was analyzed using epifluorescence (EVOS FL; AMG Life Technology). 


\section{Tube formation}

Wells of a 24-well plate were coated with $290 \mu \mathrm{l}$ of Matrigel $(10 \mathrm{mg} / \mathrm{ml})$ for $1 \mathrm{~h}$ at $37^{\circ} \mathrm{C}$. iPSC-derived endothelial cells were dissociated with TryPLE, counted and plated at 100,000 cells per well in $300 \mu \mathrm{l}$ of endothelial cell media. Transmission images were captured 1, 3, and $19 \mathrm{~h}$ post-seeding. At $21 \mathrm{~h}$ post-seeding, cells were washed 2 times in HBSS $(+\mathrm{CA} /+\mathrm{MG})$ and $8 \mu \mathrm{g} / \mathrm{ml}$ of calcein was added per well. Cells were imaged $1 \mathrm{~h}$ later at $22 \mathrm{~h}$ using epifluorescence (EVOS FL; AMG Life Technology).

\section{Electron microscopy}

Cells seeded in Matrigel for tube forming assays were fixed in one half strength Karnovsky fixative for at least $24 \mathrm{~h}$ prior to post-fixation with osmium tetraoxide (1\% in $100 \mathrm{mM}$ sodium cacodylate buffer, $\mathrm{pH} 7.2$ ). After three 20-min washes in cacodylate buffer, cells were post-stained in $2.5 \%$ uranyl acetate in cacodylate buffer and dehydrated through graded ethanol (25-100\%) prior to infiltration and embedment in Spurr's low viscosity embedding media (Electron Microscopy Sciences Cat. No. 14300). All processing steps were conducted on the tissue culture plate. After curing the resin overnight at $70^{\circ} \mathrm{C}$, resin containing cells and Matrigel was removed from the culture dishes, trimmed, and sections collected on Formvar-coated grids at a thickness of approximately $85 \mathrm{~nm}$. Images were collected on a transmission electron microscope (JEOL JEM1230).

\section{Preparation of human choroid extracellular matrix scaffolds} Human donor punches were decellularized following our previously published protocol [25]. Human donor eyes were obtained from the Iowa Lions Eye Bank (Iowa City, IA), and all experiments were performed in accordance with the Declaration of Helsinki. In brief, 6-mm $\mathrm{RPE} /$ choroid punches from healthy individuals were decellularized as follows: Punches were transferred to a 24-well plate containing $\mathrm{dH}_{2} \mathrm{O}$ and incubated at room temperature for $1.5 \mathrm{~h}, \mathrm{dH}_{2} \mathrm{O}$ was removed and replaced with $1 \%$ Triton $\mathrm{X}-100$ for $3 \mathrm{~h}$, and punches were washed 3 times for $5 \mathrm{~min}$ each in PBS followed by an overnight $18 \mathrm{~h}$ incubation in $1 \%$ Triton X-100 at $4{ }^{\circ} \mathrm{C}$. Punches were washed with PBS 3 times, and $0.1 \%$ SDS+ $0.1 \mathrm{M}$ EDTA was applied for $3 \mathrm{~h}$ at room temperature. Punches were washed 3 times with PBS and incubated with DNase I solution for $1 \mathrm{~h}$ at $37^{\circ} \mathrm{C}$ followed by 3 washes in PBS and a final rinse for $1.5 \mathrm{~h}$ in $\mathrm{dH}_{2} \mathrm{O}$.

\section{Recellularization}

Decellularized choroid punches were adhered to the bottom of a 24-well culture plate and held down by a drop of vacuum grease around the punches' edge. iPSCderived CECs were passaged using TryPLE, counted and plated at a density of 1 million cells in a 50- $\mu$ l drop of media that was placed directly on top of the punch. Cells were allowed to settle on the punch for $30 \mathrm{~min}$ prior to the addition of an additional $450 \mu \mathrm{l}$ of EC media. Cell culture media were replenished every $24 \mathrm{~h}$. Four days after plating, punches were fixed in $4 \%$ paraformaldehyde. Punches were embedded in OCT-sucrose mixture. Seven-micrometer-thick cryosections were cut from a recellularized and control block. Immunofluorescence was performed for collagen IV (Abcam ab6586) as previously described [25]. All sections were counterstained with DAPI and mounted using Aqua-Mount. Images were captured on a BX-41 Olympus microscope at $\times 40$ with a SPOT-RT camera.

\section{Results}

Differentiation of iPSC-derived choroidal endothelial cells We adopted previous publications, which use BMP-4, activin A, FGF, and VEGF to enrich for CD31positive endothelial cells [20], and modified by using a stepwise transition from mTESR $^{\mathrm{m}} 1$ to endothelial cell media with the addition of CTGF to specify choroidal endothelial cells [23]. HiPSCs were maintained on Matrigel-coated plates in mTESR $^{\text {tix }} 1$ (Fig. 2a). Colonies were gently lifted enzymatically using dispase at $80 \%$ confluency ensuring colonies were kept intact (day 0). Colonies were allowed to settle by gravity and transferred to a low bind cell culture dish to prevent adherence and promote spherical embryoid body formation (Fig. 2b-d). BMP-4, activin A, and FGF2 were added on days 1,2 , and 3 , respectively, to enrich EBs for mesoderm. On day 5, EBs were collected and allowed to settle onto a MG-coated 6-well plate at a density of $30 \mathrm{EBs}$ per well supplemented with VEGF and CTGF. VEGF was included to promote endothelial cell differentiation, and CTGF was included to promote specification of CA4-positive choroidal endothelial cells [23]. On day 6, EBs could be observed adhering to the 6-well culture dish (Fig. 2e). Over 14days, a mixed population of cells expand from the EBs with areas of cobblestone cuboidal cells (Fig. 2f-h) characteristic of ECs. When observed under epifluorescence, populations of endothelial cells positive for GFP (driven by the EC specific promoter CDH5) were observed on days 11, 12, and 13 (Fig. 3a-f) of differentiation which morphologically resemble the cobblestone morphology of HUVECS, and an immortalized choroidal endothelial cell line isolated from a human donor eye (Fig. 3g). On day 14 , the heterogenous cell population was placed under zeocin selection to isolate a $\mathrm{CDH} 5$-enriched population. Alternatively, the cell monolayer was enzymatically dissociated using TryPLE and CD31-positive endothelial cells were isolated with anti-CD31 MACS 


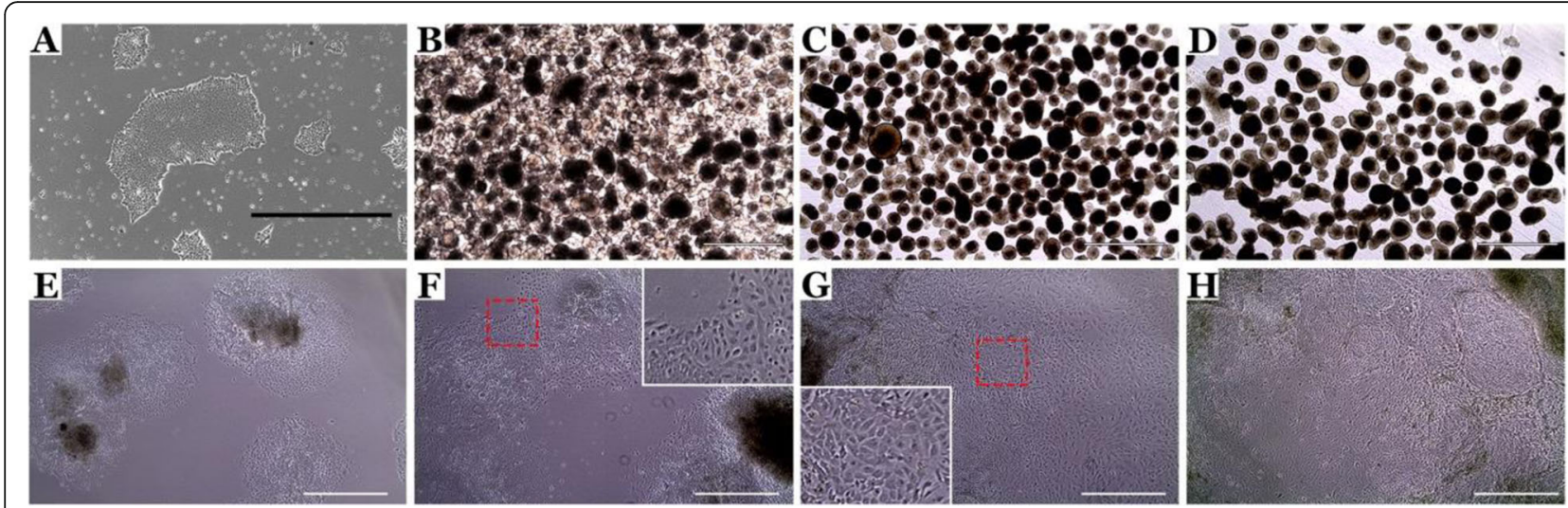

Fig. 2 Differentiation of induced pluripotent stem cells into choroidal endothelial cells. Representative light microscope images acquired during the stepwise differentiation of induced pluripotent stem cells into mesoderm-derived choroidal endothelial cells. (a) iPSC colonies are expanded prior to differentiation and embryoid body (EB) formation. EBs were kept in suspension for 5 days. EB appearance after 1 (b), 3 (c), and 5 days (d) post-formation. On day 5, EBs were adhered to a Matrigel-coated 6-well plate at a density of 30 EBs per well. After 24 h (i.e., differentiation day 6), EBs have stuck to the Matrigel-coated surface (e). EBs gave rise to a heterogenous population of cells over 14 days. Areas of EC with cobblestone morphology can be seen at days $7(\mathbf{f}), 12(\mathbf{g})$, and $14(\mathbf{h})$. Note that red square in $(\mathbf{f})$ and $(\mathbf{g})$ demarcates the area depicted in the corresponding high-magnification inlay. Scale bars $=1000 \mu \mathrm{M}$

beads. The zeocin selection and CD31 bead enrichment protocols were compared in subsequent immunocytochemical and functional assays.

\section{Characterization of ECs}

iPSC-derived ECs labeled positively for the classical endothelial cell marker CD31 (Fig. 4a, e and Additional file 2) following expansion, and we observed 96.74\% positivity post-MACS sort and $95.80 \%$ positivity postzeocin selection (Table 1). Furthermore, our iPSC- derived ECs express the markers CA4 (Fig. 4c, g and Additional file 2) and RGCC (Fig. 4d, h and Additional file 2), which, as compared to endothelial cells lining arteries and veins, are both highly abundant in choriocapillaris endothelial cells (Fig. 4i). $88.90 \%$ of cells stained positively for CA4 post-MACS sort and $88.98 \%$ stained positively post-zeocin selection while $95.01 \%$ of cells were positive for RGCC following MACS sort and 95.78\% were positive following zeocin selection. We compared iPSC-derived CECs to a primary immortalized

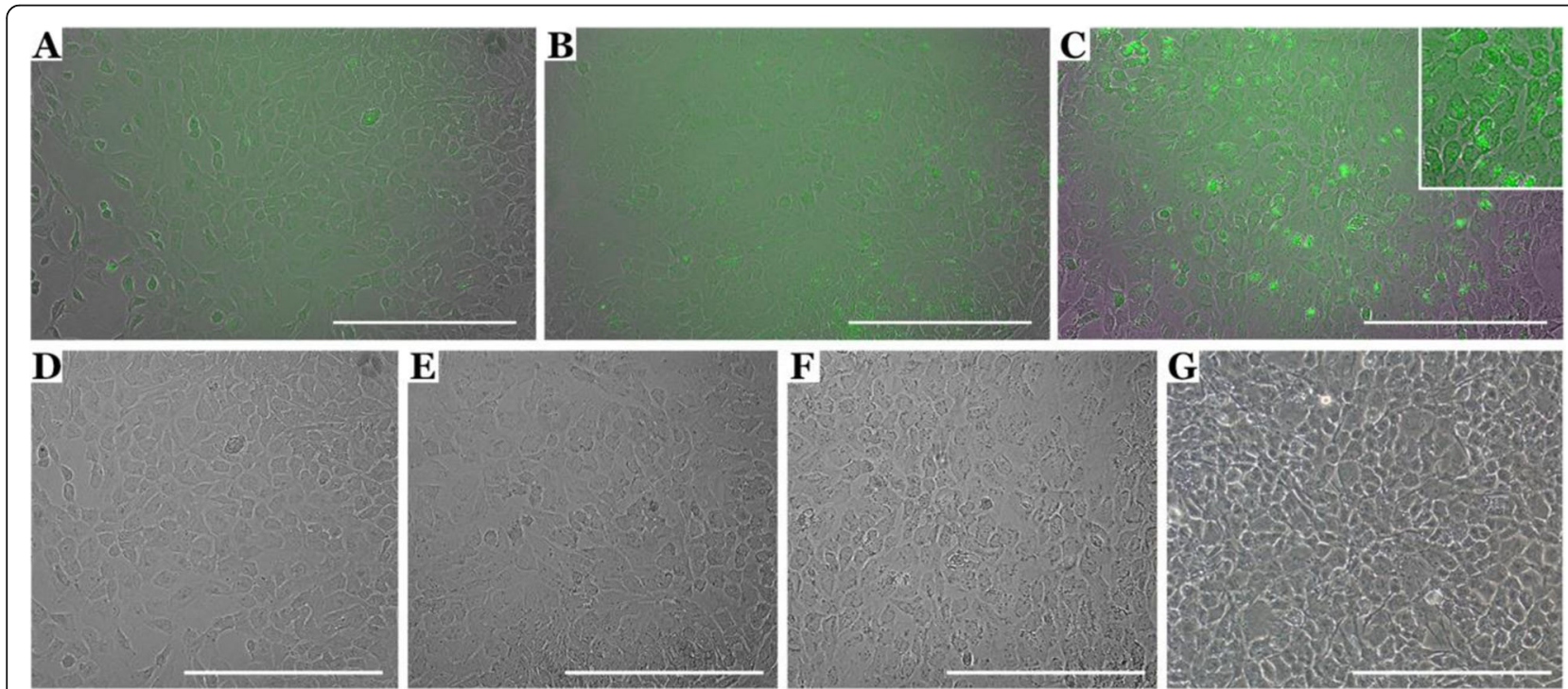

Fig. 3 CDH5-GFP-positive cells are observed at day 11 of differentiation. (a) CDH5-GFP expressing cells are observed on day 11 (a, d), day 12 (b, e), and day 13 $(\mathbf{c}, \mathbf{f})$ during stepwise differentiation accompanied by a characteristic cobblestone morphology ( $\mathbf{d}-\mathbf{f}$ ) which closely resembles choroidal endothelial cells immortalized from a healthy human donor eye $(\mathbf{g})$. Scale bars $=400 \mu \mathrm{M}$. Note that inlay in (c) is a high-magnification image taken from the same field 


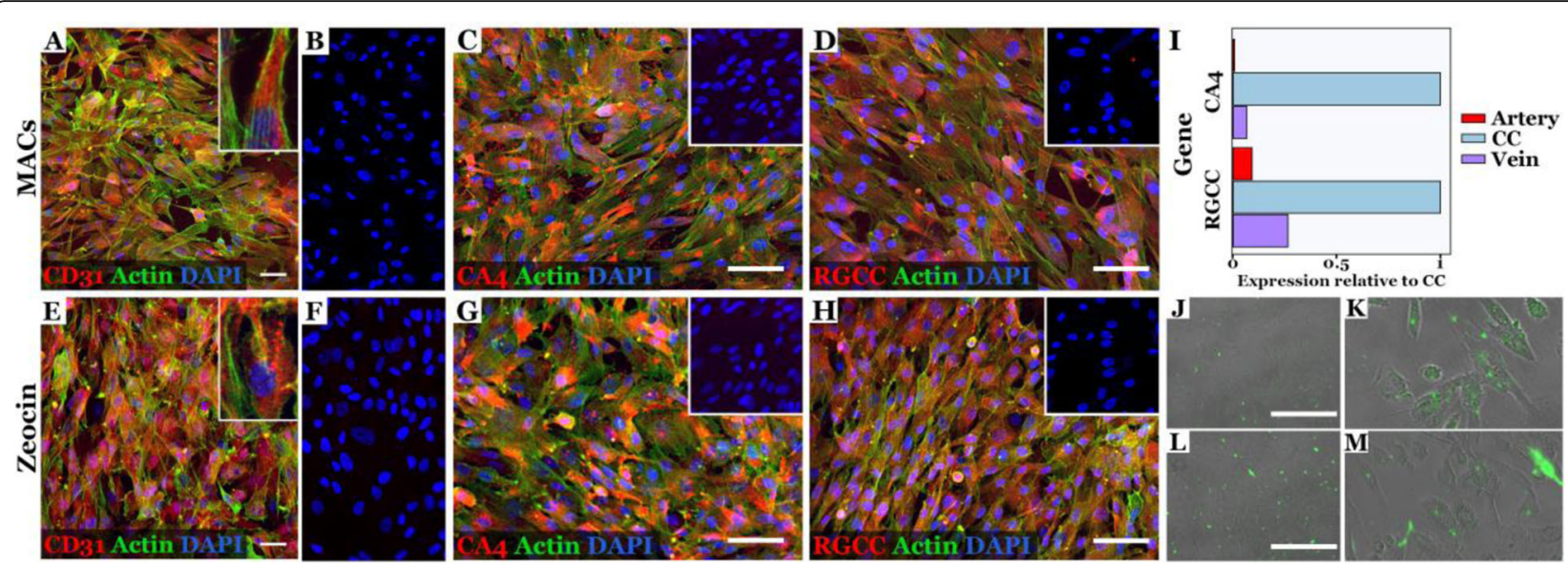

Fig. 4 iPSC-derived choroidal endothelial cells express CD31, CA4, and RGCC. Representative immunocytochemical staining of endothelial cellspecific markers CD31 (a, e red), CA4 (c, g red), and RGCC (d, $\mathbf{h}$ red) in differentiated cells post-endothelial cell enrichment via CD31-positive MACS sorting (a-d) or CDH5 lentiviral zeocin selection (e-h). Positivity was determined via comparison to secondary antibody-only controls (b, $\mathbf{f}$ and inserts in top right corner). Cells were co-stained for actin (green), and nuclei were counterstained with DAPI. Scale bars = 50 $\mu$ M. (i) CA4 and RGCC expression are enriched in primary human choriocapillaris endothelial cells (CC) opposed to primary human arterial (Artery) and venous (Vein) choroidal endothelial cells [26]. To calculate the relative expression of each gene compared to the choriocapillaris cluster (x-axis), the average expression of CA4 and RGCC were calculated for each cell type and divided by the mean expression in the choriocapillaris cluster. Alexa Fluro 488-labeled acetylated LDL uptake in iPSC-derived choroidal endothelial cells post-CD31 MACS sort (j, $\mathbf{k})$ and zeocin selection (I, m)

human donor CEC line and observed similar staining patterns for CD31, CA4, and RGCC using epifluorescence microscopy (Additional file 3).

\section{Characterization of EC function}

The internalization of acetylated low-density lipoprotein (ac-LDL) and Matrigel tube formation assays are commonly used to assess EC function in vitro. In addition to expression of specific cell surface markers, iPSC-derived ECs were able to uptake ac-LDL (Fig. $4 j-m$ ) and form tubes similar to primary human choroidal ECs in Matrigel (Additional file 3) following either MACS (Fig. 5a-d) or zeocin selection (Fig. 5e-h). Electron microscopy of the tubes indicated the tubes contain a lumen (Fig. 5i), suggesting that these cells have the ability to participate in angiogenesis. Decellularized human donor choroidal extracellular matrix allows us to test the ability of iPSCderived CECs to migrate into and repopulate empty choroidal vessels. We observed multiple CECs in empty choroidal vessels (Fig. 5, note presence of DAPI $(k, l)$ and expression of CD31 (l; high mag inlay) in donor
iPSC-CECs) compared to a control punch in which no iPSCs were added (Fig. 5j, lack of DAPI-positive native cells).

\section{Discussion}

iPSC technology allows us to generate endothelial cells from patient fibroblasts, providing a valuable resource for studying patient-specific ECs in vitro. In addition, patient-derived ECs could be a valuable resource for cell replacement to repair damaged vessels. Successful cell replacement strategies are organ specific and likely require the transplantation of ECs that closely resemble those that are lost in disease. In the eye, the ECs which line the choriocapillaris are unique in their structure and function; therefore, to accurately model retinal degenerative diseases such as AMD, we require a protocol to reliably produce CECs from iPSCs.

Our group previously published a method to produce murine CECs via co-culture [27], and furthermore, using RNA-Seq identified CTGF as a key factor promoting CEC-like differentiation in human iPSCs [23]. In our

Table 1 Percent of cells expressing each marker following selection

\begin{tabular}{llllll}
\hline & Marker & Percent Positive C1 & Percent Positive C2 & Percent Positive C3 & Mean positive \\
\hline MACS & CD31 & 95.93 & 97.73 & 96.55 & 96.74 \\
& CA4 & 90.97 & 88.89 & 86.84 & 88.90 \\
& RGCC & 96.99 & 96.88 & 91.18 & 95.01 \\
Zeo & CD31 & 96.84 & 96.55 & 94.00 & 95.80 \\
& CA4 & 87.94 & 87.50 & 91.49 & 88.98 \\
& RGCC & 97.18 & 95.16 & 95.00 & 95.78 \\
\hline
\end{tabular}




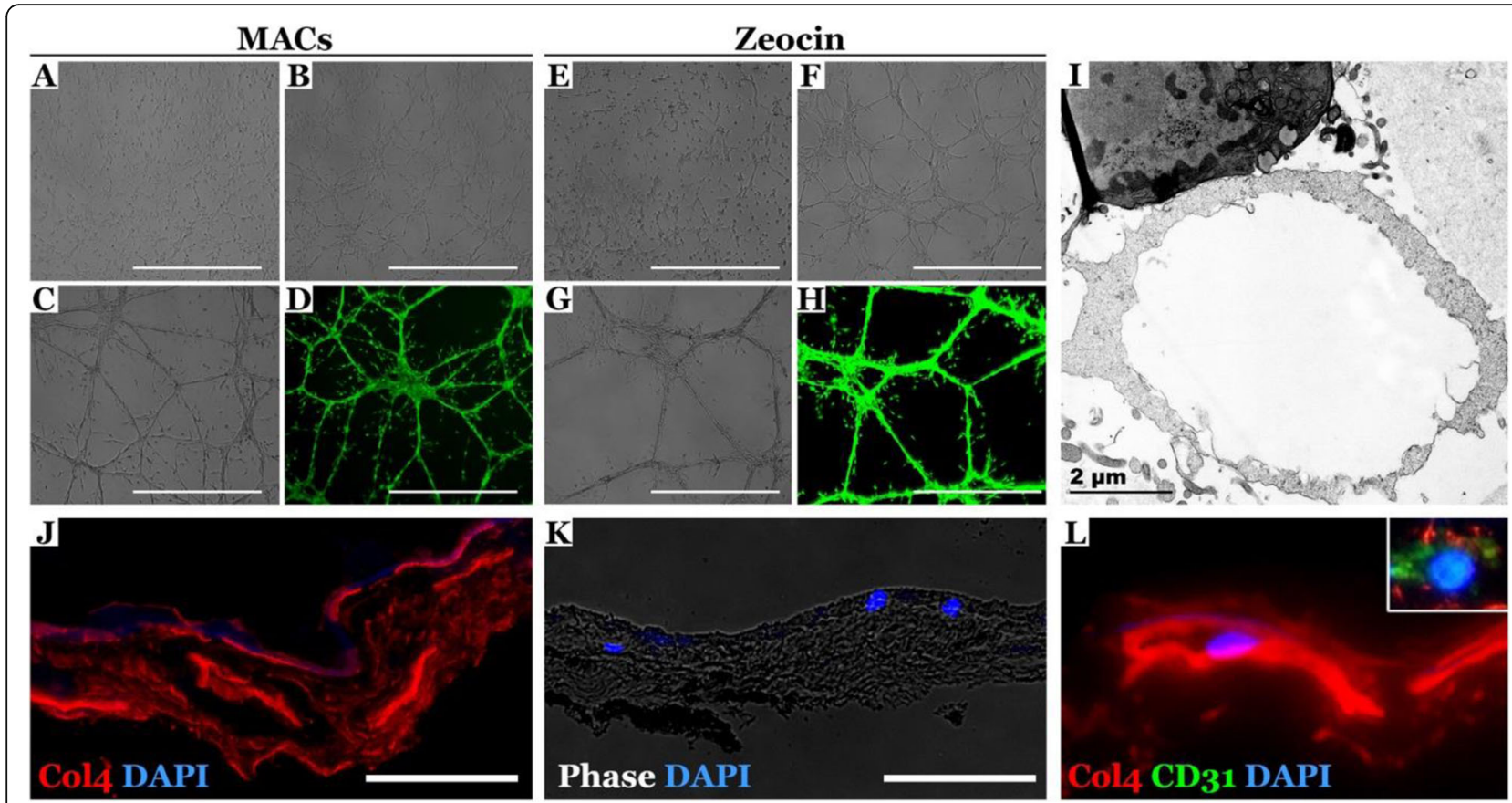

Fig. 5 iPSC-derived choroidal endothelial cells form vascular tubes in Matrigel and can migrate into decellularized human choroid extracellular matrix. Microscopy images demonstrate formation of vasculature $1(\mathbf{a}, \mathbf{e}), 3(\mathbf{b}, \mathbf{f}), 19(\mathbf{c}, \mathbf{g})$, and $22 \mathrm{~h}(\mathbf{d}, \mathbf{h})$ post-seeding of iPSC-derived CECs into a 3D Matrigel matrix (a-d): CD31 MACS sorted cells; $(\mathbf{e}-\mathbf{h})$ : zeocin selected cells. Calcein was used at the 22-h time point $(\mathbf{d}$, $\mathbf{h})$ to demonstrate cell viability. Scale bars $=1000 \mu \mathrm{M}$. (i) Transmission electron micrograph of iPSC-derived CEC tube demonstrates lumen formation. (j) Immunofluorescence labeling of human RPE/choroid after decellularization. Anti-collagen IV (red) highlights the structure of the choroid tissue following decellularization, and the absence of nuclear DAPI (blue) demonstrates the absence of native endothelial cells. IPSC-derived CECs are observed within the vascular tubes of the choriocapillaris $(\mathbf{k}, \mathbf{l})$ in both a brightfield and anti-collagen IV labeled image

previous study, we employed spontaneous differentiation of EBs to generate mesoderm precursors, followed by the addition of CTGF to direct mesoderm differentiation toward CECs. Early endothelial cell protocols utilized spontaneous differentiation of EBs to obtain ECs [18]. However, significant increases in EC yield were achieved by using a directed approach. This involves the addition of growth factors important in specifying mesoderm during embryonic development, including BMP-4 and FGF, which derive from the epiblast, and activin A, which derives from the hypoblast $[19,20]$. Adding these factors during EB formation can steer germ layer differentiation toward a mesoderm cell fate, which in turn leads to increased yield of EC differentiation. In order to improve CEC differentiation to ensure reproducible results in iPSCs derived from multiple donors, we adopted a directed approach. Prior to differentiation, we transduced 3 iPSC donor lines with a lentiviral reporter. This lentivirus contains $\mathrm{CDH} 5$ driving GFP with zeocin resistance, allowing us to track EC differentiation, while also providing a unique selection method for iPSCderived ECs. We opted to use EBs for our first step of differentiation and slowly transitioned aggregating iPSC colonies from $\mathrm{mTESR}^{\mathrm{m} 1} 1$ to endothelial cell medium with stepwise addition of BMP-4, activin A, and FGF-2
(Fig. 1). On day 5, EBs were adhered to a Matrigelcoated plate. We added VEGF and CTGF to promote differentiation of choroidal endothelial cells. Previously, we have reported that CTGF is sufficient to drive CECs [23]. CTGF is a matricellular protein that is important for vessel growth during retinal development [28] and has been shown to promote endothelial cell proliferation, migration, and tube formation in vitro [29]. On day 11, we observed GFP-positive subpopulations of cells, indicating a commitment to an endothelial cell fate. On day 14, we observed a heterogeneous population of cell types. In order to purify endothelial cells, we adopted two independent positive selection methods. The first takes advantage of the zeocin selection cassette in our reporter lentivirus. Placing cells under zeocin selection for $48 \mathrm{~h}$, we saw drastic cell death of non-CDH5-positive cells. Alternatively, we tested whether we could purify endothelial cells using a non-lentiviral method. We choose to use CD31-positive MACS beads to magnetically separate CD31-expressing endothelial cells from other cell types. We observed that approximately $7 \%$ of overall cells were CD31 positive.

Following both selection methods, we expanded the endothelial cells and used immunohistochemistry to verify expression of CD31. Regardless of selection method, 
we obtained pure populations of cells expressing this classical endothelial cell marker. Although both selection methods gave rise to cells that express endothelial cell markers, they do appear slightly different morphologically. Specifically, CD31 selected cells look more endothelial like, retaining a cobblestone morphology. In contrast, cells selected with zeocin appear more elongate and fibroblast like. Zeocin causes cell death by inducing double-stranded DNA breaks and is therefore harder on the cells. It is likely that only the most robust endothelial cells that are more proliferative survive zeocin selection.

As indicated above, choriocapillaris loss occurs early in the pathogenesis of AMD, often preceding degeneration of the overlying RPE and photoreceptor cells [810]. As such, autologous iPSC-derived CEC replacement early in the disease course could be advantageous in slowing AMD progression, including the atrophic dry form of the disease, which currently has no treatment. AMD patients with a polymorphism in the $C F H$ gene $(\mathrm{Y} 402 \mathrm{H})$ have increased risk of CEC loss [30]. In order to restore healthy CECs, iPSC CRISPR technology could be used to restore wildtype $\mathrm{CFH}$, and these CRISPR-corrected iPSCs would then be differentiated to CECs to be used for cell transplantation. For such a strategy, the best approach for deriving iPSC-CECs would be to utilize the CD31 MACS selection method, as it does not require lentiviral integration and produces cells which are morphologically superior.

In order to determine whether our EC population reassembled ECs in the choriocapillaris, we immunolabeled for carbonic anhydrase IV (CA4), a specific marker for ECs of the choroid capillaries. CA4 is visible immunohistochemically during development of the choroidal vasculature [31] and is observed in the choriocapillaris but not in the larger vessels in Sattler's or Haller's layers in adulthood [32]. Our differentiation protocol produced ECs positive for CA4, suggesting successful reassembly of ECs in the choriocapillaris. Recently, our group has identified an additional marker, regulator of cell cycle gene (RGCC), that is useful for distinguishing endothelial cells in the choriocapillaris from ECs lining arteries or veins [26]. Like CA4, RGCC is more abundant in choroid capillary ECs than other ECs in the choroid. In addition to CA4 positivity, we found that our iPSC-ECs stain positively for RGCC providing strong evidence that we obtained choriocapillaris-specific ECs.

A hallmark of ECs is the ability to form capillary tubelike networks. Here, we show that iPSC-derived ECs organized into 3-dimensional tube networks in Matrigel. Using electron microscopy, we confirm the presence of vascular lumens within the tubular network. To determine whether iPSC-derived CECs could be used for cell replacement strategies, we tested their ability to migrate into and repopulate empty choriocapillaris vessels.
Decellularization of human donor RPE/choroid creates an empty ECM scaffold [25]. We observed that iPSC-derived CECs can revascularize the empty choriocapillaris tubes. This will begin to allow us to develop cell replacement strategies for age-related macular degeneration, which has a hallmark feature of early choroidal endothelial cell loss. Thus, we have developed a stepwise protocol to successfully generate iPSC-derived CECs that morphologically and structurally resemble ECs of the choriocapillaris.

\section{Conclusions}

In summary, we have developed a stepwise differentiation protocol to generate patient-specific choroidal endothelial cells. We have demonstrated two methods to purify CECs post-differentiation, making this protocol translatable to multiple laboratory settings. We have provided a tool to successfully study CECs in vitro for disease modeling in AMD and other choroid-related ocular diseases.

\section{Supplementary information}

Supplementary information accompanies this paper at https://doi.org/10. 1186/s13287-020-01903-4.

Additional file 1. Detailed list of reagents, equipment and step by step protocol for differentiation of choroidal endothelial cells.

Additional file 2. iPSC-derived choroidal endothelial cells express CD31, CA4 and RGCC.

Additional file 3. Comparison between IPSC-derived choroidal endothelial cells and a primary human choroidal endothelial cell line for expression of CD31, CA4 and RGCC

Additional file 4. Human primary choroidal endothelial cell line is capable of forming tubes in a 3D Matrigel matrix.

\section{Abbreviations}

ECs: Endothelial cells; CEC: Choroidal endothelial cell; AMD: Age-related macular degeneration; iPSCs: Induced pluripotent stem cells;

CTGF: Connective tissue growth factor; RPE: Retinal pigment epithelium; RGCC: Regulator of cell cycle; CA4: Carbonic anhydrase IV

\section{Acknowledgements}

The RGCC monoclonal antibody developed by JHU/CDI was obtained from the Developmental Studies Hybridoma Bank, created by the NICHD of the $\mathrm{NIH}$, and maintained at the University of lowa, Department of Biology, lowa City, IA 52242

The data presented herein were obtained at the Flow Cytometry Facility, which is a Carver College of Medicine/Holden Comprehensive Cancer Center core research facility at the University of lowa. The facility is funded through user fees and the generous financial support of the Carver College of Medicine, Holden Comprehensive Cancer Center, and lowa City Veteran's Administration Medical Center.

\section{Authors' contributions}

KM conceived and performed the experiments and wrote the manuscript. JCG, APV, and DO performed the experiments and edited the manuscript. MJR performed the transmission electron microscopy. ICH wrote the manuscript. EMS, RFM, and BAT conceived and supervised the experiments, provided the funding, and wrote the manuscript. The authors read and approved the final manuscript. 


\section{Funding}

This research was funded by the National Institutes of Health, grants EY024605 and EY-025580, and the Elmer and Sylvia Sramek Charitable Foundation.

\section{Availability of data and materials}

The datasets supporting the conclusions of this article are included within the article and its additional files.

\section{Ethics approval and consent to participate}

Patient-derived skin biopsies were collected under informed consent following IRB approval. Biopsies were used for fibroblast isolation and iPSC generation.

\section{Consent for publication}

Not applicable.

\section{Competing interests}

The authors declare that they have no competing interests.

Received: 8 May 2020 Revised: 12 August 2020

Accepted: 25 August 2020 Published online: 23 September 2020

\section{References}

1. Muller AM, Hermanns MI, Skrzynski C, Nesslinger M, Muller KM, Kirkpatrick CJ. Expression of the endothelial markers PECAM-1, vWf, and CD34 in vivo and in vitro. Exp Mol Pathol. 2002;72(3):221-9.

2. Aird WC. Phenotypic heterogeneity of the endothelium: I. Structure, function, and mechanisms. Circ Res. 2007;100(2):158-73.

3. Rosa S, Praca C, Pitrez PR, Gouveia PJ, Aranguren XL, Ricotti L, et al. Functional characterization of iPSC-derived arterial- and venous-like endothelial cells. Sci Rep. 2019:9(1):3826.

4. Gage PJ, Rhoades W, Prucka SK, Hjalt T. Fate maps of neural crest and mesoderm in the mammalian eye. Invest Ophthalmol Vis Sci. 2005;46(11): 4200-8.

5. Hayreh SS. Segmental nature of the choroidal vasculature. $\mathrm{Br} J$ Ophthalmol. 1975;59(11):631-48.

6. Hayreh SS. Physiological anatomy of the choroidal vascular bed. Int Ophthalmol. 1983;6(2):85-93.

7. Hayreh SS. Posterior ciliary artery circulation in health and disease: the Weisenfeld lecture. Invest Ophthalmol Vis Sci. 2004;45(3):749-57 8

8. Mullins RF, Johnson MN, Faidley EA, Skeie JM, Huang J. Choriocapillaris vascular dropout related to density of drusen in human eyes with early age-related macular degeneration. Invest Ophthalmol Vis Sci. 2011;52(3):1606-12.

9. Whitmore SS, Braun TA, Skeie JM, Haas CM, Sohn EH, Stone EM, et al. Altered gene expression in dry age-related macular degeneration suggests early loss of choroidal endothelial cells. Mol Vis. 2013;19:2274-97.

10. Sohn EH, Flamme-Wiese MJ, Whitmore SS, Workalemahu G, Marneros AG, Boese EA, et al. Choriocapillaris degeneration in geographic atrophy. Am J Pathol. 2019;189(7):1473-80.

11. Kogure K, Choromokos E. Infrared absorption angiography. J Appl Physiol. 1969:26(1):154-7.

12. Yannuzzi LA, Slakter JS, Sorenson JA, Guyer DR, Orlock DA. Digital indocyanine green videoangiography and choroidal neovascularization. Retina. 1992;12(3):191-223.

13. Ahn SM, Lee SY, Hwang SY, Kim SW, Oh J, Yun C. Retinal vascular flow and choroidal thickness in eyes with early age-related macular degeneration with reticular pseudodrusen. BMC Ophthalmol. 2018;18(1):184.

14. Braun PX, Mehta N, Gendelman I, Alibhai AY, Moult EM, Zhao Y, et al. Global analysis of macular choriocapillaris perfusion in dry age-related macular degeneration using swept-source optical coherence tomography angiography. Invest Ophthalmol Vis Sci. 2019;60(15):4985-90.

15. Choi W, Moult EM, Waheed NK, Adhi M, Lee B, Lu CD, et al. Ultrahigh-speed, swept-source optical coherence tomography angiography in nonexudative age-related macular degeneration with geographic atrophy. Ophthalmology. 2015;122(12):2532-44.

16. Thulliez M, Zhang Q, Shi Y, Zhou H, Chu Z, de Sisternes L, et al. Correlations between choriocapillaris flow deficits around geographic atrophy and enlargement rates based on swept-source OCT imaging. Ophthalmol Retina. 2019;3(6):478-88
17. Cicinelli MV, Rabiolo A, Marchese A, de Vitis L, Carnevali A, Querques L, et al. Choroid morphometric analysis in non-neovascular age-related macular degeneration by means of optical coherence tomography angiography. Br J Ophthalmol. 2017;101(9):1193-200.

18. Levenberg S, Golub JS, Amit M, Itskovitz-Eldor J, Langer R. Endothelial cells derived from human embryonic stem cells. Proc Natl Acad Sci U S A. 2002; 99(7):4391-6.

19. Goldman O, Feraud O, Boyer-Di Ponio J, Driancourt C, Clay D, Le BousseKerdiles MC, et al. A boost of BMP4 accelerates the commitment of human embryonic stem cells to the endothelial lineage. Stem Cells. 2009;27(8): $1750-9$

20. James D, Nam HS, Seandel M, Nolan D, Janovitz T, Tomishima M, et al. Expansion and maintenance of human embryonic stem cell-derived endothelial cells by TGFbeta inhibition is Id1 dependent. Nat Biotechnol. 2010;28(2):161-6.

21. Nourse MB, Halpin DE, Scatena M, Mortisen DJ, Tulloch NL, Hauch KD, et al. VEGF induces differentiation of functional endothelium from human embryonic stem cells: implications for tissue engineering. Arterioscler Thromb Vasc Biol. 2010;30(1):80-9.

22. Zhang J, Chu LF, Hou Z, Schwartz MP, Hacker T, Vickerman V, et al. Functional characterization of human pluripotent stem cell-derived arterial endothelial cells. Proc Natl Acad Sci U S A. 2017:114(30):E6072-e8.

23. Songstad AE, Worthington KS, Chirco KR, Giacalone JC, Whitmore SS, Anfinson KR, et al. Connective tissue growth factor promotes efficient generation of human induced pluripotent stem cell-derived choroidal endothelium. Stem Cells Transl Med. 2017:6(6):1533-46.

24. Giacalone JC, Miller MJ, Workalemahu G, Reutzel AJ, Ochoa D, Whitmore SS, et al. Generation of an immortalized human choroid endothelial cell line (iChEC-1) using an endothelial cell specific promoter. Microvasc Res. 2019; 123:50-7.

25. Chirco KR, Worthington KS, Flamme-Wiese MJ, Riker MJ, Andrade JD, Ueberheide BM, et al. Preparation and evaluation of human choroid extracellular matrix scaffolds for the study of cell replacement strategies. Acta Biomater. 2017;57:293-303.

26. Voigt AP, Mulfaul K, Mullin NK, Flamme-Wiese MJ, Giacalone JC, Stone EM, et al. Single-cell transcriptomics of the human retinal pigment epithelium and choroid in health and macular degeneration. Proc Natl Acad Sci U S A. 2019;116(48):24100-7.

27. Songstad AE, Wiley LA, Duong K, Kaalberg E, Flamme-Wiese MJ, Cranston CM, et al. Generating iPSC-derived choroidal endothelial cells to study age-related macular degeneration. Invest Ophthalmol Vis Sci. 2015;56(13):8258-67.

28. Pi L, Xia H, Liu J, Shenoy AK, Hauswirth WW, Scott EW. Role of connective tissue growth factor in the retinal vasculature during development and ischemia, Invest Ophthalmol Vis Sci. 2011:52(12):8701-10.

29. Shimo T, Nakanishi T, Nishida T, Asano M, Kanyama M, Kuboki T, et al. Connective tissue growth factor induces the proliferation, migration, and tube formation of vascular endothelial cells in vitro, and angiogenesis in vivo. J Biochem. 1999:126(1):137-45.

30. Mullins RF, Schoo DP, Sohn EH, Flamme-Wiese MJ, Workamelahu G, Johnston RM, et al. The membrane attack complex in aging human choriocapillaris: relationship to macular degeneration and choroidal thinning. Am J Pathol. 2014;184(11):3142-53.

31. Baba T, Grebe R, Hasegawa T, Bhutto I, Merges C, McLeod DS, et al. Maturation of the fetal human choriocapillaris. Invest Ophthalmol Vis Sci. 2009:50(7):3503-11.

32. Hageman GS, Zhu XL, Waheed A, Sly WS. Localization of carbonic anhydrase IV in a specific capillary bed of the human eye. Proc Natl Acad Sci U S A. 1991;88(7):2716-20.

\section{Publisher's Note}

Springer Nature remains neutral with regard to jurisdictional claims in published maps and institutional affiliations. 